\title{
Revisiting the hot matter in the center of gamma-ray bursts and supernovae
}

\author{
Ang $\mathrm{Li}^{1,2}$ and Tong $\mathrm{Liu}^{1,2}$ \\ ${ }^{1}$ Department of Astronomy and Institute of Theoretical Physics and Astrophysics, Xiamen University, Xiamen, 361005 Fujian, \\ PR China \\ e-mail: liang@xmu.edu.cn \\ 2 State Key Laboratory of Theoretical Physics, Institute of Theoretical Physics, Chinese Academy of Sciences, 100190 Beijing, \\ PR China
}

Received 15 April 2013 / Accepted 11 May 2013

\begin{abstract}
Aims. Hot matter with nucleons can be produced in the inner region of the neutrino-dominated accretion flow in gamma-ray bursts or during the proto-neutron star birth in successful supernovae. The composition and equation of state of the matter depend on the dynamic $\beta$ equilibrium under various neutrino opacities. The strong interaction between nucleons may also play an important role. We plan to extend the previous studies by incorporating these two aspects in our model.

Methods. The modification of the $\beta$-equilibrium condition from neutrino optically thin to thick was modeled by an equilibrium factor $\chi$ ranging between the neutrino-freely-escaping case and the neutrino-trapped case. We employed the microscopic BruecknerHartree-Fock approach extended to the finite temperature regime to study the interacting nucleons.

Results. We show the composition and chemical potentials of the hot nuclear matter for different densities and temperatures at each stage of $\beta$ equilibrium. We also compare our realistic equation of states with those of the free-gas model. We find that it is important to properly describe the neutrino opacity and the strong interaction between nucleons, and they should be taken into account in model calculations.
\end{abstract}

Key words. equation of state - gamma-ray burst: general - neutrinos - nuclear reactions, nucleosynthesis, abundances dense matter - supernovae: general

\section{Introduction}

Gamma-ray bursts (GRBs) and supernovae (SNs) are extremely powerful explosions in the universe. Hot dense matter may be generated in the centers of these objects. The properties of the matter, such as its composition and equation of state (EoS), are very important for studies of GRBs and SNs.

First, for the central engines of GRBs, one of the plausible candidates is the neutrino-dominated accretion flow (NDAF) around a rotating stellar-mass black hole. Such systems may stem from the merger of two compact objects or a collapsar. The NDAF model has been widely applied to explaining the energy source and several observations of GRBs in the past decade (e.g., Popham et al. 1999; Narayan et al. 2001; Kohri \& Mineshige 2002; Di Matteo et al. 2002; Kohri et al. 2005; Lee et al. 2005; Gu et al. 2006; Chen \& Beloborodov 2007; Janiuk et al. 2007; Kawanaka \& Mineshige 2007; Lei et al. 2009; Liu et al. 2007, 2008, 2010a,b, 2012a,b, 2013; Sun et al. 2012; Kawanaka \& Kohri 2012; Kawanaka et al. 2013). Hot matter approaching nuclear densities $\left(\rho \sim 10^{10}-10^{13} \mathrm{~g} \mathrm{~cm}^{-3}\right.$ and $\left.T \sim 10^{10}-10^{11} \mathrm{~K}\right)$ may appear in the inner regions of those disks, so a proper description of them should be included in the NDAF model. Liu et al. (2007) studied the radial structure and the neutrino annihilation luminosity of the NDAF. They arbitrarily introduced a bridging formula to treat the radial distribution of the electron fraction between neutrino optically thin and thick limits, thanks to an analytical relation of the chemical potential equilibrium obtained for the former case by Yuan (2005). But they ignored the strong interaction between nucleons, and simplified the calculations by suggesting that the matter was in a free-gas state, which essentially meant that there was no difference between the number density and the energy density. Kawanaka \& Mineshige (2007) paid attention to the difference in the number density and the energy density, but an active connection between neutrino optically thin and thick limits was not included in their calculations. Therefore, a more improved theoretical NDAF model should be presented, especially for the inner region of a disk.

Second, SNs (or collapsars) have also been widely calculated or simulated in the past decade (see, e.g., MacFadyen et al. 2001; Proga et al. 2003; Buras et al. 2006; Burrows et al. 2007; Iwakami et al. 2008; Hammer et al. 2010). In a successful SN, the birth of a proto-neutron star may go through several distinct steps (see, e.g., Prakash et al. 2001). The first step lasts less than one minute, during which the star with a neutrino-trapped core of mass experiences the core bounce and the passage of a shock through the star's mantle. The outer mantle is both accreting matter from the surrounding area and losing energy due to the thermal neutrino emission. In the second step, the accretion is no longer important and neutrino-cooling dominates. During the above two steps, a hot dense state $\left(\rho \sim 10^{12}-10^{14} \mathrm{~g} \mathrm{~cm}^{-3}\right.$ and $\left.T \sim 10^{10} \mathrm{~K}\right)$ is present both in the core and in the outer part. In such a state the neutrino production exists along with their transportation, so the dynamics of the production process should play an important role in calculating and simulating of SNs, where the opacity has to be taken into account. 
Finally, because neutrino radiation is the main cooling mechanism for the hot matter of GRBs and SNs, the chemical potential equilibrium in the matter may depend on the neutrino opacity, namely the dynamics of the $\beta$ processes (Imshennik et al. 1967). Also, nucleons may interact with each other in such dense matter, thus an improved EoS including the strong interaction becomes imperative. In this paper, we then focus on the effects of the dynamics of the $\beta$ processes and of the strong interaction on various input microphysics of GRBs and SNs, such as the relative composition and the EoS of the matter.

Accordingly, we assume that the nuclei are dissolved completely into nucleons, therefore nuclear many-body theories are applicable to deriving nucleonic chemical potentials. Incorporating the strong interaction between nucleons will certainly affect the constituent chemical potentials, the composition, and the EoS of the matter. The employed nuclear model is the microscopic Brueckner-Hartree-Fock (BHF) approach widely used for studying dense stellar matter and neutron star properties (Baldo et al. 1997; Baldo 1999; Baldo \& Ferreira 1999; Burgio et al. 2003; Zuo et al. 2004; Li et al. 2006, 2010; Nicotra et al. 2006; Peng et al. 2008; Burgio et al. 2011; Chen et al. 2013), as we discuss in Sect. 2.2. We stress that our aim is not to model the centers of GRBs and SNs, but instead to explore how the properties of the hot matter in the center depend on the strong-interaction effect and the dynamic $\beta$-process related to neutrinos. Such effects are usually missing in most of the GRB and SN studies.

The paper is organized as follows. In Sect. 2, we establish our physical model and describe the numerical methods for the calculation in detail. In Sect. 3, numerical results are presented. We present our main conclusions in Sect. 4.

\section{Model}

\section{1. $\beta$ equilibrium of the hot nuclear matter}

For the hot matter where neutrinos are completely trapped, the system can achieve its equilibrium via the following $\beta$ processes,

$$
\begin{aligned}
& \mathrm{e}^{-}+\mathrm{p} \rightleftharpoons \mathrm{n}+v_{\mathrm{e}} \\
& \mathrm{e}^{+}+\mathrm{n} \rightleftharpoons \mathrm{p}+\bar{v}_{\mathrm{e}} \\
& \mathrm{n} \rightleftharpoons \mathrm{p}+\mathrm{e}^{-}+\bar{v}_{\mathrm{e}}
\end{aligned}
$$

The reaction rates of the $\beta$ reactions are equal to those of the corresponding inverse processes. Because the photons are also trapped (the chemical potential of photons $\mu_{\gamma}=0$ ), the chemical equilibria $\gamma+\gamma \rightleftharpoons \mathrm{e}^{+}+\mathrm{e}^{-} \rightleftharpoons \nu_{\mathrm{e}}+\bar{v}_{\mathrm{e}}$ give

$\mu_{\mathrm{e}^{-}}=-\mu_{\mathrm{e}^{+}}$.

Then by writing $\mu_{\mathrm{e}^{-}} \equiv \mu_{\mathrm{e}}$, the usual chemical equilibrium condition can be applied as

$\mu_{\mathrm{n}}+\mu_{v_{\mathrm{e}}}=\mu_{\mathrm{p}}+\mu_{\mathrm{e}}$,

or

$\mu_{\mathrm{n}}=\mu_{\mathrm{p}}+\mu_{\mathrm{e}}$,

if the chemical potential of the trapped neutrinos is zero. These are the well-known chemical equilibrium conditions that are generally used to determine the composition of the hot matter under $\beta$ equilibrium (Nicotra et al. 2006; Peng et al. 2008; Li et al. 2010; Burgio et al. 2011; Chen et al. 2013).
However, if neutrinos can leave the system freely, the $\beta$-equilibrium of the $\beta$ reactions cannot be treated as a chemical equilibrium problem. In such circumstance the steady state of the hot matter is achieved under the following condition,

$\lambda_{\mathrm{e}^{-} \mathrm{p}}=\lambda_{\mathrm{e}^{+} \mathrm{n}}+\lambda_{\mathrm{n}}$

where $\lambda_{\mathrm{e}^{-} p}, \lambda_{\mathrm{e}^{+} n}$ stands for the rate of the $\mathrm{e}^{ \pm}$-captures,

$\mathrm{e}^{-}+\mathrm{p} \rightarrow \mathrm{n}+v_{\mathrm{e}}$,

$\mathrm{e}^{+}+\mathrm{n} \rightarrow \mathrm{p}+\bar{v}_{\mathrm{e}}$,

and $\lambda_{\mathrm{n}}$ is the rate of neutron decay,

$\mathrm{n} \rightarrow \mathrm{p}+\mathrm{e}^{-}+\bar{v}_{\mathrm{e}}$

The reaction rates $\lambda$ should be functions of the temperature $T$ and the constituent chemical potentials. Compared with the rate of positron capture by neutrons $\lambda_{\mathrm{e}^{+} \mathrm{n}}$, the rate of neutron decay $\lambda_{\mathrm{n}}$ in this case could be neglected, therefore the $\beta$-equilibrium condition is reduced to $\lambda_{\mathrm{e}^{-} \mathrm{p}}=\lambda_{\mathrm{e}^{+} \mathrm{n}}$ (Beloborodov 2003; Pruet et al. 2003; Yuan 2005), and finally

$\mu_{\mathrm{n}}=\mu_{\mathrm{p}}+2 \mu_{\mathrm{e}}$

after the used assumption of the elastic approximation (e.g., Yuan 2005). Again zero chemical potential is applied for neutrinos.

By combining Eqs. (6) and (11) we introduce an equilibrium factor $\chi$ in the range of $[1,2]$, to explore the effect of the dynamics of the $\beta$ processes, namely

$\mu_{\mathrm{n}}=\mu_{\mathrm{p}}+\chi \mu_{\mathrm{e}}$,

where $\chi=1$ suggests completely neutrino-trapped matter, and $1<\chi \leqslant 2$ corresponds to the matter with a certain amount of freely escaping neutrinos.

To find the composition and the EoS of the matter, for each baryon number density $n_{\mathrm{B}}$, Eq. (12) should be solved together with the charge neutrality condition,

$n_{\mathrm{e}^{-}}-n_{\mathrm{e}^{+}}=n_{\mathrm{p}}$,

and the conservation of the baryon number,

$n_{\mathrm{n}}+n_{\mathrm{p}}=n_{\mathrm{B}}$.

The nucleonic chemical potentials are derived from the free energy density of the nuclear matter, based on the finitetemperature BHF nuclear many-body approach (as illustrated below in Sect. 2.2). The chemical potentials of the noninteracting leptons $\mathrm{e}^{ \pm}$are obtained by solving numerically the free Fermi gas model at a finite temperature. Specifically, the number density of species $i\left(i=\mathrm{e}^{ \pm}\right)$is written as

$n_{i}(T)=\int_{0}^{\infty} \frac{g}{(2 \pi \hbar)^{3}} n_{i}^{\mathrm{F}}(k, T) \mathrm{d}^{3} k$,

where $(2 \pi \hbar)^{3}$ is the "unit" volume of a cell in the phase space and $g$ is the number of states of a particle with a given momentum $k$. For $\mathrm{e}^{ \pm}$leptons, $g$ equals 2 . Finally, $n_{i}^{\mathrm{F}}(k, T)$ is the FermiDirac-statistic,

$$
\begin{aligned}
& n_{\mathrm{e}^{-}}^{\mathrm{F}}(k, T)=\frac{1}{\exp \left[\left(E(k)-\mu_{\mathrm{e}}\right) / k_{\mathrm{B}} T\right]+1}, \\
& n_{\mathrm{e}^{+}}^{\mathrm{F}}(k, T)=\frac{1}{\exp \left[\left(E(k)+\mu_{\mathrm{e}}\right) / k_{\mathrm{B}} T\right]+1},
\end{aligned}
$$


Ang Li and Tong Liu: The hot matter in GRBs and SNs

where $k_{\mathrm{B}}$ is the Boltzmann constant and the energy $E(k)=$ $\sqrt{k^{2}+m_{\mathrm{e}}^{2}}$ with $m_{\mathrm{e}}$ being the mass of the electrons or positrons.

Once the nucleonic and leptonic chemical potentials are determined, one can proceed to calculate the composition of the hot $\beta$-equilibrium matter by solving Eqs. (13) and (14), together with Eq. (12). Then the total energy density $\rho$ and the total pressure $P$ of the system are

$\rho=\rho_{\mathrm{l}}+\rho_{\mathrm{B}}$,

$P=P_{1}+P_{\mathrm{B}}$,

where $\rho_{1}$ and $P_{1}$ are the standard contributions of the leptons:

$\rho_{1}=\frac{8 \pi}{(2 \pi \hbar)^{3}} \int_{0}^{\infty}\left(f_{\mathrm{e}^{-}}-f_{\mathrm{e}^{+}}\right) E(k) k^{2} \mathrm{~d} k$

$P_{1}=\frac{8 \pi}{3(2 \pi \hbar)^{3}} \int_{0}^{\infty}\left(f_{\mathrm{e}^{-}}-f_{\mathrm{e}^{+}}\right) k^{4} / E(k) \mathrm{d} k$.

We present in the following section how the contributions of baryons $\left(\rho_{\mathrm{B}}, P_{\mathrm{B}}\right)$ are determined.

\subsection{BHF nuclear many-body approach}

Currently, one of the most advanced microscopic approaches to the EoS of the nuclear matter is the BHF model (Baldo 1999). Recently, this model has been extended to the finite-temperature regime within the Bloch-De Dominicis formalism (Bloch \& De Dominicis 1958, 1959a,b). The central quantity of the BHF formalism is the $G$-matrix, which in the finite-temperature extension (Bloch \& De Dominicis 1958, 1959a,b; Baldo 1999; Baldo \& Ferreira 1999) is determined by solving numerically the Bethe-Goldstone equation, and can be written in operatorial form as

$G_{a b}[W]=V_{a b}+\sum_{c} \sum_{p, p^{\prime}} V_{a c}\left|p p^{\prime}\right\rangle \frac{Q_{c}}{W-E_{c}+\mathrm{i} \epsilon}\left\langle p p^{\prime}\right| G_{c b}[W]$

where the indices $a, b, c$ indicate pairs of nucleons and the Pauli operator $Q$ and energy $E$ determine the propagation of intermediate nucleon pairs. In a given nucleon-nucleon channel $c=(12)$ one has

$Q_{(12)}=\left[1-n_{1}^{\mathrm{F}}\left(k_{1}\right)\right]\left[1-n_{2}^{\mathrm{F}}\left(k_{2}\right)\right]$,

$E_{(12)}=m_{1}+m_{2}+e_{1}\left(k_{1}\right)+e_{2}\left(k_{2}\right)$,

with the single-particle (s.p.) energy $e_{i}(k)=k^{2} / 2 m_{i}+U_{i}(k)$, the above-mentioned Fermi distribution $n_{i}^{\mathrm{F}}(k)=\left(\mathrm{e}^{\left[e_{i}(k)-\tilde{\mu}_{i}\right] / T}+1\right)^{-1}$, the starting energy $W$, and the two-body interaction (bare potential) $V$ as fundamental input. The various s.p. potentials within the continuous choice are given by

$U_{1}\left(k_{1}\right)=\operatorname{Re} \sum_{2=\mathrm{n}, \mathrm{p}} \sum_{k_{2}} n\left(k_{2}\right)\left\langle k_{1} k_{2}\left|G_{(12)(12)}\left[E_{(12)}\right]\right| k_{1} k_{2}\right\rangle_{A}$,

where $k_{i}$ generally denote momentum and spin. For given partial densities $n_{i}(i=\mathrm{n}, \mathrm{p})$ and temperature $T$, Eqs. (22)-(25) have to be solved self-consistently along with the equations for the auxiliary chemical potentials $\tilde{\mu}_{i}, n_{i}=\int_{k} n_{i}^{\mathrm{F}}(k)$.

Regarding the interactions, we use the Argonne $V_{18}$ nucleonnucleon potential (Wiringa et al. 1995) together with the microscopic nuclear three-body forces (TBF; Grangé et al. 1989; Lejeune et al. 2000; Zuo et al. 2002). The including of TBF accomplishes excellently two important tasks. First, the corresponding zero-temperature nuclear EoS reproduces the nuclear matter saturation point correctly and fulfills several requirements

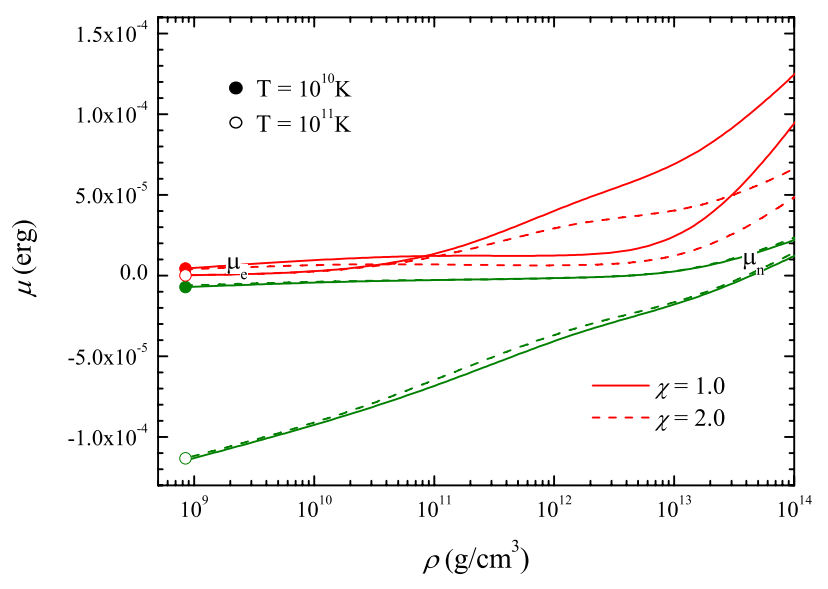

Fig. 1. Chemical potentials of both electrons and neutrons (red lines for $\mu_{\mathrm{e}}$, and green ones for $\mu_{\mathrm{n}}$ ) as a function of the energy density $\rho$ at two fixed temperatures $T=10^{10} \mathrm{~K}$ (filled symbol) and $T=10^{11} \mathrm{~K}$ (open symbol), for both $\chi=1$ (solid lines) and $\chi=2$ (dashed lines) cases, respectively.

from the nuclear phenomenology (Baldo et al. 1997). Second, the main relativistic effect can be taken into account, and the results in our nonrelativistic scheme agree well with the predictions of the corresponding relativistic approaches (Zuo et al. 2002).

Once the different s.p. potentials for the species $i=\mathrm{n}, \mathrm{p}$ are known, the free energy density of nuclear matter has the simplified expression

$f=\sum_{i}\left[\sum_{k} n_{i}^{\mathrm{F}}(k)\left(\frac{k^{2}}{2 m_{i}}+\frac{1}{2} U_{i}(k)\right)-T s_{i}\right]$,

where

$s_{i}=-\sum_{k}\left(n_{i}^{\mathrm{F}}(k) \ln n_{i}^{\mathrm{F}}(k)+\left[1-n_{i}^{\mathrm{F}}(k)\right] \ln \left[1-n_{i}^{\mathrm{F}}(k)\right]\right)$

is the entropy density for component $i$ treated as a free gas with s.p. spectrum $e_{i}(k)$ (Baldo 1999; Baldo \& Ferreira 1999).

All thermodynamic quantities of interest can then be computed from the free energy density, Eq. (26); namely, the "true" chemical potentials $\mu_{i}(i=\mathrm{n}, \mathrm{p})$, internal energy density $\rho_{\mathrm{B}}$, and pressure $P_{\mathrm{B}}$ are

$$
\begin{aligned}
\mu_{i} & =\frac{\partial f}{\partial n_{i}}, \\
s & =-\frac{\partial f}{\partial T}, \\
\rho_{\mathrm{B}} & =f+T s, \\
P_{\mathrm{B}} & =n^{2} \frac{\partial(f / n)}{\partial n}=\sum_{i} \mu_{i} n_{i}-f .
\end{aligned}
$$

\section{Results}

For both GRBs and SNs, a hot state with nucleons exists with $\rho \sim 10^{9}-10^{14} \mathrm{~g} \mathrm{~cm}^{-3}$ and $T \sim 10^{9}-10^{11} \mathrm{~K}$. We adopted these parameter ranges in our model. One additional parameter $\chi$, the so-called equilibrium factor, is introduced to incorporate the effect of the neutrino opacity, and its value should be between 1 and 2, as discussed in the previous section.

We first display in Fig. 1 the chemical potentials of both electrons and neutrons as a function of the energy density $\rho$ 


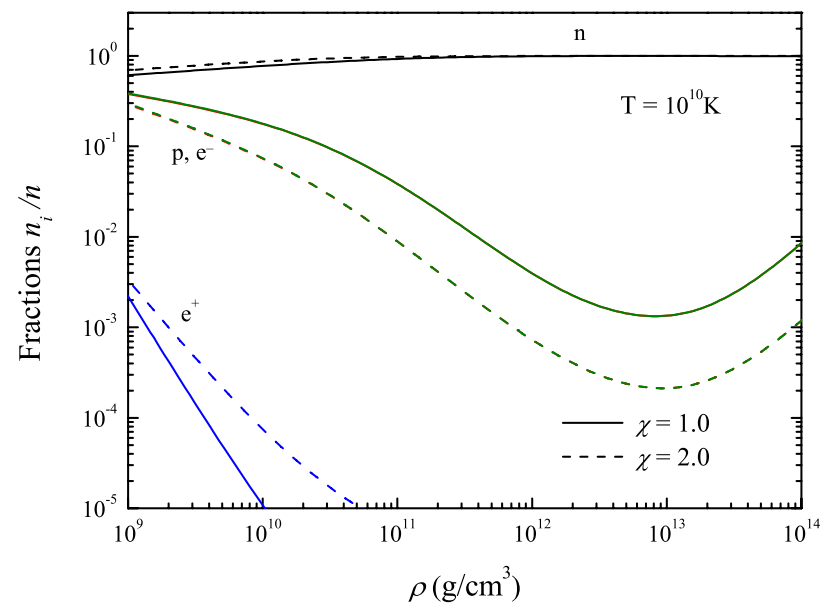

Fig. 2. Relative fractions $n_{i} / n_{\mathrm{B}}\left(i=\mathrm{n}, \mathrm{p}, \mathrm{e}^{-}, \mathrm{e}^{+}\right)$as a function of the energy density $\rho$ at fixed temperature $T=10^{10} \mathrm{~K}$, for both $\chi=1$ (solid lines) and $\chi=2$ (dashed lines) cases.

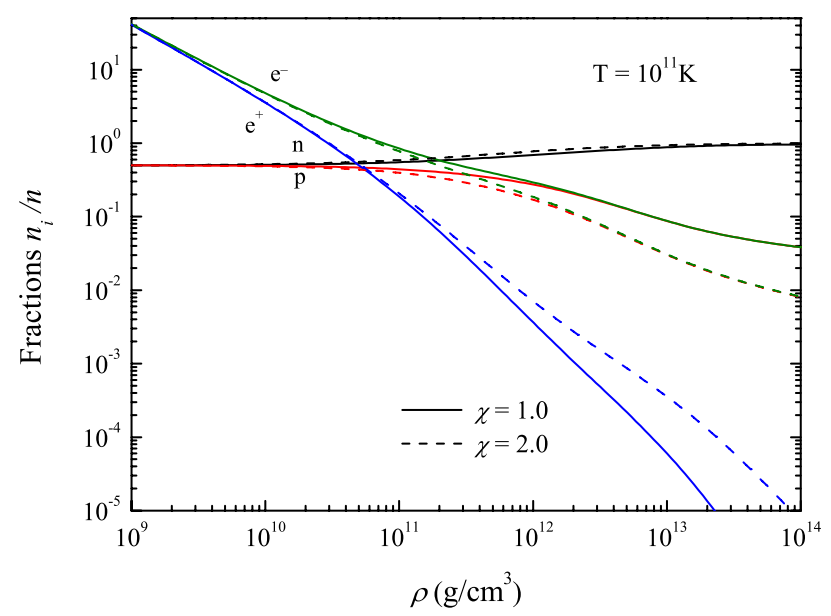

Fig. 3. Same as Fig. 2, but for $T=10^{11} \mathrm{~K}$.

at two fixed temperatures $T=10^{10} \mathrm{~K}$ and $T=10^{11} \mathrm{~K}$, for both $\chi=1$ and $\chi=2$ cases. Regardless of the temperature, the electron chemical potentials are always positive and increase monotonously with the density. Since electrons are treated as a degenerate fermi gas, the corresponding degenerate pressures are expected to increase with the density as well. However, $\mu_{\mathrm{n}}$ changes its sign from negative to positive at high densities (around $10^{14} \mathrm{~g} \mathrm{~cm}^{-3}$ ), which simply means that the strong interaction dominates for such dense matter. We mention here that the nuclear saturation density is about $2.5 \times 10^{14} \mathrm{~g} \mathrm{~cm}^{-3}$. Moreover, we find that the equilibrium factor $\chi$ affects $\mu_{\mathrm{n}}$ only slightly. Its influence on $\mu_{\mathrm{e}}$ is evident, but mainly in the high-density region. With the increase in the $\chi$ parameter, the electron chemical potential $\mu_{\mathrm{e}}$ is largely reduced, which means there is a reduced lepton fraction in the matter. Also, compared with the low temperature $\left(T=10^{10} \mathrm{~K}\right)$ case, we find much rapid increases in both $\mu_{\mathrm{e}}$ and $\mu_{n}$ at high temperature $\left(T=10^{11} \mathrm{~K}\right)$ case.

Those results should have significant impacts on the study of the NDAF model, since we know that there is a wide range of density in the inner region of the disk (see, e.g., Liu et al. 2007, 2008), $\rho \sim 10^{9}-10^{13} \mathrm{~g} \mathrm{~cm}^{-3}$, and for a typical accretion rate of $1 M_{\odot} \mathrm{s}^{-1}$, the neutrino optical depth would change from thick to thin if one moves away from the central black hole. Therefore, one should include the dependence of the constituent chemical
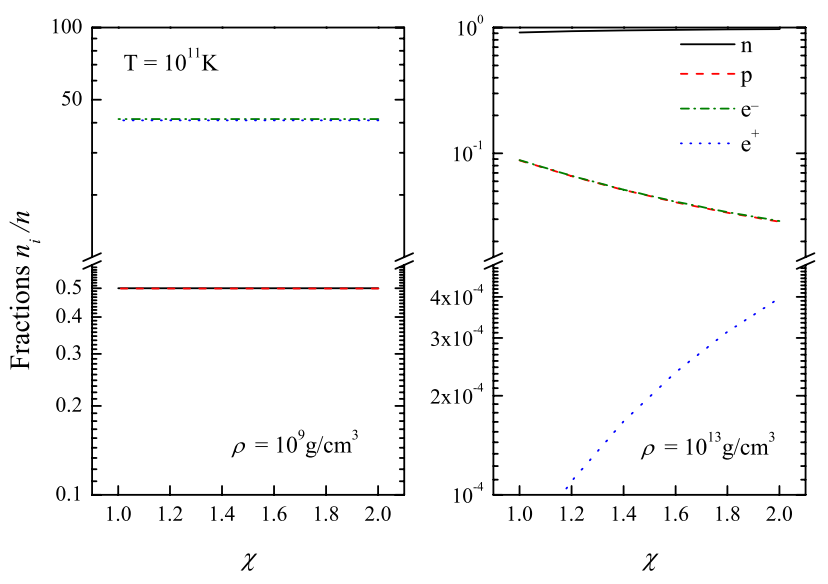

Fig. 4. Relative fractions $n_{i} / n_{\mathrm{B}}\left(i=\mathrm{n}, \mathrm{p}, \mathrm{e}^{-}, \mathrm{e}^{+}\right)$as a function of $\chi$ parameter at two fixed densities $\rho=10^{9} \mathrm{~g} \mathrm{~cm}^{-3}$ (left panel) and $10^{13} \mathrm{~g} \mathrm{~cm}^{-3}$ (right panel) for $T=10^{11} \mathrm{~K}$.

potentials on the equilibrium condition, the temperature, and the energy density, as demonstrated in the above figure.

We then show the compositions of the matter in Fig. 2 (for $T=10^{10} \mathrm{~K}$ ) and Fig. 3 (for $T=10^{11} \mathrm{~K}$ ). The relative fractions $n_{i} / n_{\mathrm{B}}\left(i=\mathrm{n}, \mathrm{p}, \mathrm{e}^{-}, \mathrm{e}^{+}\right)$are plotted as a function of the energy density $\rho$, for both the $\chi=1$ and $\chi=2$ cases. In the lowtemperature case of $T=10^{10} \mathrm{~K}$ (Fig. 2), the proton fractions are very similar to the electron fractions, and they both increase with the density as a combined result of an increased electron chemical potential $\mu_{\mathrm{e}}$ and the charge neutrality condition (Eq. (13)). In contrast, the positron fractions decrease very quickly with increasing density as a natural result of the Fermi-Dirac distribution employed for the leptons. Here neutrons compose most of the matter (more than $\sim 70 \%$ ), and the matter is practically $n p \mathrm{e}^{-}$ for the chosen temperature $T=10^{10} \mathrm{~K}$. If the matter is much hotter, for example $T=10^{11} \mathrm{~K}$ in Fig. 3, at relatively low density (below $10^{11} \mathrm{~g} \mathrm{~cm}^{-3}$ ), a large number of $\mathrm{e}^{ \pm}$pairs exist regardless of the choice of $\chi$ parameter. This means the efficient creation of the $\mathrm{e}^{ \pm}$pair is the characteristic of a hot nuclear system.

Figure 4 shows the compositions as a function of $\chi$ parameter at two fixed densities $\rho=10^{9} \mathrm{~g} \mathrm{~cm}^{-3}$ (left panel) and $10^{13} \mathrm{~g} \mathrm{~cm}^{-3}$ (right panel) for a fixed temperature of $T=10^{11} \mathrm{~K}$. At low densities around $10^{9} \mathrm{~g} \mathrm{~cm}^{-3}$, the variation in the equilibrium parameter $\chi$ only trivially affects the relative fractions, as one might also notice in Fig. 3. But at high densities the $\chi$ dependence becomes important, so the accretion matter in the inner region of the disk should be more appropriately modeled in the NDAF model.

Finally we summarize our results of EoSs in Fig. 5 for two temperatures $T=10^{10} \mathrm{~K}$ and $T=10^{11} \mathrm{~K}$, and for both $\chi=1$ and $\chi=2$ cases. The predictions of the free-gas model are also shown for comparison. In both models, the higher temperature, the stiffer EoS. Because electrons contribute equally to our model and the free-gas model, the differences between the thick and thin lines arise from the difference in the nucleon part. Our realistic EoSs usually lie below the ones based on the free-gas approximation because it is the long-range gravity that dominates in the matter, not the short-range nucleon-nucleon force, except at high densities. The strong interaction cannot be ignored in such dense matter.

As commonly recognized, the pressure from nucleons should dominate in the inner region of the NDAF when the mass accretion rate is greater than $0.01 M_{\odot} \mathrm{s}^{-1}$ (see, e.g., Chen \& Beloborodov 2007; Liu et al. 2007; Kawanaka \& Mineshige 2007). As shown above, the nucleonic EoS is subject to change 
Ang Li and Tong Liu: The hot matter in GRBs and SNs

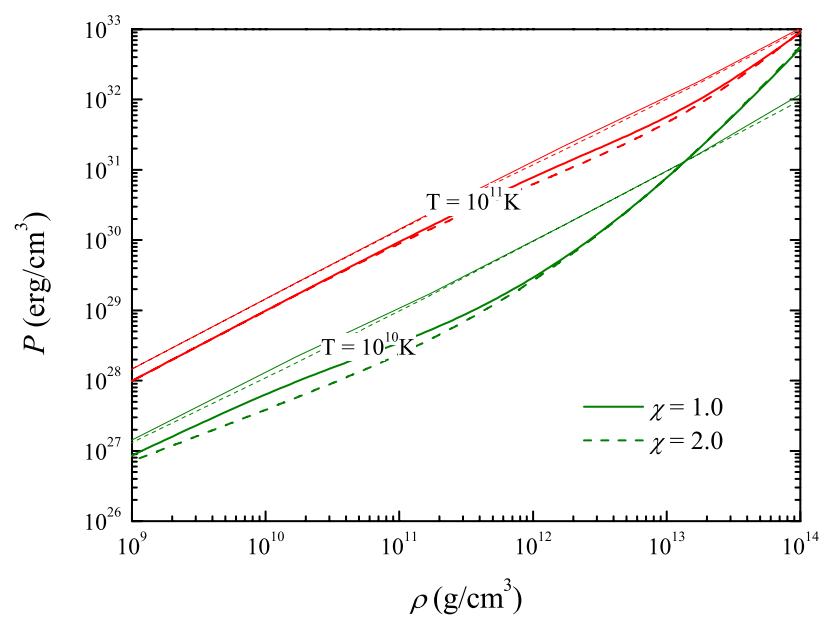

Fig. 5. EoSs for two temperatures $T=10^{10} \mathrm{~K}$ (red lines) and $T=10^{11} \mathrm{~K}$ (green lines), and for both $\chi=1$ (solid lines) and $\chi=2$ (dashed lines) cases. The predictions of free gas model are also shown in thin lines for comparison.

if we include more microscopic physics beyond the simple standard free-gas model. Such a simple model is widely used in the NDAF or collapsar models (see, e.g., Popham et al. 1999; MacFadyen et al. 2001; Chen \& Beloborodov 2007; Liu et al. 2007). For an improved study, a detailed database of the resulting pressure with the change in the temperature, the density, and the neutrino opacity should be built.

\section{Conclusion}

In this paper, various properties of the hot nuclear matter possible in the inner regions of GRBs and SNs have been revisited. We employed the microscopic BHF approach to account for the strong interaction between nucleons, and calculated the nucleonic chemical potentials and the nucleonic EoS in this method. We introduced a parameterized chemical potential equilibrium between neutrino optically thin and thick limits, and showed the compositions and the EoSs of the matter at several temperatures under different chemical potential equilibria, labeled by the socalled equilibrium factor $\chi$. We also compared of the EoSs with those of the free-gas model. We find that the effect of the neutrino opacity and the strong interaction can be very important for the description of the hot matter. They should be taken into account in future model calculations.

For example, since one popular central engine model that powers GRBs consists of a black hole and an NDAF with a hypercritical mass accretion rate, and the neutrino annihilation luminosity in the NDAF model can be significantly affected by the matter properties of the inner region of the disk, we will revisit the NDAF model by incorporating the findings of the present work in the description of the inner region, to verify more convincingly whether the NDAF model still can be one of the candidates of the central engines of GRBs.
Acknowledgements. We would like to thank Prof. T. T. Fang who carefully read the manuscript and made valuable suggestions. This work was supported by the National Basic Research Program (973 Program) of China under Grant 2009CB824800 and the National Natural Science Foundation of China under grants 11103015 and 11233006.

\section{References}

Baldo, M. 1999, Nuclear Methods and the Nuclear Equation of State (Singapore, World Scientific), Int. Rev. Nucl. Phys., 8

Baldo, M., \& Ferreira, L. S. 1999, Phys. Rev. C, 59, 682

Baldo, M., Bombaci, I., \& Burgio, G. F. 1997, A\&A, 328, 274

Beloborodov, A. M. 2003, ApJ, 588, 931

Bloch, C., \& De Dominicis, C. 1958, Nucl. Phys., 7, 459

Bloch, C., \& De Dominicis, C. 1959a, Nucl. Phys., 10, 181

Bloch, C., \& De Dominicis, C. 1959b, Nucl. Phys., 10, 509

Buras, R., Janka, H.-T., Rampp, M., \& Kifonidis, K. 2006, A\&A, 457, 281

Burgio, F., Schulze, H. J., \& Weber, F. 2003, A\&A, 408, 675

Burgio, F., Schulze, H. J., \& Li, A. 2011, Phys. Rev. C, 83, 025804

Burrows, A., Dessart, L., Livne, E., Ott, C. D., \& Murphy, J. 2007, ApJ, 664, 416

Chen, H., Burgio, F., Schulze, H. J., \& Yasutake, N. 2013, A\&A, 551, A13

Chen, W.-X., \& Beloborodov, A. M. 2007, ApJ, 657, 383

Di Matteo, T., Perna, R., \& Narayan, R. 2002, ApJ, 579, 706

Grangé, P., Lejeune, A., Martzolff, M., \& Mathiot, J.-F. 1989, Phys. Rev. C, 40, 1040

Gu, W.-M., Liu, T., \& Lu, J.-F. 2006, ApJ, 643, L87

Hammer, N. J., Janka, H.-T., \& Müller, E. 2010, ApJ, 714, 1371

Imshennik, S., Nadezhin, D. K., \& Pinaev, V. S. 1967, Sov. Astron., 10, 970

Iwakami, W., Kotake, K., Ohnishi, N., Yamada, S., \& Sawada, K. 2008, ApJ, 678, 1207

Janiuk, A., Yuan, Y., Perna, R., \& Di Matteo, T. 2007, ApJ, 664, 1011

Kawanaka, N., \& Kohri, K. 2012, MNRAS, 419, 713

Kawanaka, N., \& Mineshige, S. 2007, ApJ, 662, 1156

Kawanaka, N., Piran, T., \& Krolik, J. H. 2013, ApJ, 766, 31

Lee, W. H., Ramirez-Ruiz, E., \& Page, D. 2005, ApJ, 632, 421

Lei, W. H., Wang, D. X., Zhang, L., et al. 2009, ApJ, 700, 1970

Lejeune, A., Lombardo, U., \& Zuo, W. 2000, Phys. Lett. B, 477, 45

Li, A., Burgio, G. F., Lombardo, U., \& Zuo, W., 2006, Phys. Rev. C, 74, 055801

Li, A., Zhou, X. R., Burgio, F., \& Schulze, H. J. 2010, Phys. Rev. C, 81, 025806

Liu, T., Gu, W.-M., Xue, L., \& Lu, J.-F. 2007, ApJ, 661, 1025

Liu, T., Gu, W.-M., Xue, L., Weng, S.-S., \& Lu, J.-F. 2008, ApJ, 676, 545

Liu, T., Gu, W.-M., Dai, Z.-G., \& Lu, J.-F. 2010a, ApJ, 709, 851

Liu, T., Liang, E.-W., Gu, W.-M., et al. 2010b, A\&A, 516, A16

Liu, T., Gu, W.-M., Xue, L., \& Lu, J.-F. 2012a, Ap\&SS, 337, 711

Liu, T., Liang, E.-W., Gu, W.-M., et al. 2012b, ApJ, 760, 63

Liu, T., Xue, L., Gu, W.-M., \& Lu, J.-F. 2013, ApJ, 762, 102

Kohri, K., \& Mineshige, S. 2002, ApJ, 577, 311

Kohri, K., Narayan, R., \& Piran, T. 2005, ApJ, 629, 341

MacFadyen, A. I., Woosley, S. E., \& Heger, A. 2001, ApJ, 550, 410

Narayan, R., Piran, T., \& Kumar, P. 2001, ApJ, 557, 949

Nicotra, O. E., Baldo, M., Burgio, F., \& Schulze, H. J. 2006, A\&A, 451, 213

Peng, G.-X., Li, A., \& Lombardo, U. 2008, Phys. Rev. C, 77, 065807

Popham, R., Woosley, S. E., \& Fryer, C. 1999, ApJ, 518, 356

Prakash, M., Lattimer, J. M., Pons, J. A., Steiner, A. W., \& Reddy, S. 2001, Physics of Neutron Star Interiors, 578, 364

Proga, D., MacFadyen, A. I., Armitage, P. J., \& Begelman, M. C. 2003, ApJ, 599, L5

Pruet, J., Woosley, S. E., \& Hoffman, R. D. 2003, ApJ, 586, 1254

Sun, M.-Y., Liu, T., Gu, W.-M., \& Lu, J.-F. 2012, ApJ, 752, 31

Wiringa, R. B., Stoks, V. G. J., \& Schiavilla, R. 1995, Phys. Rev. C, 51, 38

Yuan, Y.-F. 2005, Phys. Rev. D, 72, 013007

Zuo, W., Lejeune, A., Lombardo, U., \& Mathiot, J.-F. 2002, Eur. Phys. J. A, 14, 469

Zuo, W., Li, A., Li, Z.-H., \& Lombardo, U., 2004, Phys. Rev. C, 70, 055802 